\title{
Phonated Speech Reconstruction Using Twin Mapping Models
}

\author{
Hamid R. Sharifzadeh*, Amir HajiRassouliha*, Ian V. McLoughlin ${ }^{\dagger}$, Iman T. Ardekani*, Jacqueline E. Allen $\ddagger$ \\ *Signal Processing Lab, Unitec Institute of Technology, Auckland, New Zealand \\ Email: \{hsharifzadeh, ahajirassouliha, iardekani\}@unitec.ac.nz \\ ${ }^{\dagger}$ School of Computing, The University of Kent, Kent, United Kingdom \\ Email: i.v.mcloughlin@kent.ac.uk \\ $\ddagger$, Department of Otolaryngology, North Shore Hospital, Auckland, New Zealand \\ Email: jeallen@voiceandswallow.co.nz
}

\begin{abstract}
Computational speech reconstruction algorithms have the ultimate aim of returning natural sounding speech to aphonic and dysphonic individuals. These algorithms can also be used by unimpaired speakers for communicating sensitive or private information. When the glottis loses function due to disease or surgery, aphonic and dysphonic patients retain the power of vocal tract modulation to some degree but they are unable to speak anything more than hoarse whispers without prosthetic aid. While whispering can be seen as a natural and secondary aspect of speech communications for most people, it becomes the primary mechanism of communications for those who have impaired voice production mechanisms, such as laryngectomees.

In this paper, by considering the current limitations of speech reconstruction methods, a novel algorithm for converting whispers to normal speech is proposed and the efficiency of the algorithm is discussed. The proposed algorithm relies upon twin mapping models and makes use of artificially generated whispers (called whisperised speech) to regenerate natural phonated speech from whispers. Through a training-based approach, the mapping models exploit whisperised speech to overcome frame to frame time alignment problem in the speech reconstruction process.
\end{abstract}

\section{INTRODUCTION}

The human voice is the most magnificent instrument for communication, capable of expressing deep emotions, conveying oral history through generations, or of starting a war. However, those who suffer from aphonia (no voice) and dysphonia (voice disorders) are unable to make use of this critical form of communication. They are typically unable to project anything more than hoarse whispers [1].

Whispered speech is useful for quiet and private communications in daily life [2], [3], [4]. Unimpaired speakers occasionally use whispers to communicate in the public locations such as libraries, cinema theatres, or during lectures and meetings. But whispered speech becomes the primary communicative mechanism for many people experiencing voice box difficulties [5], [6]. There is no definitive estimate of the global population suffering some form of voice problem, but information from a number of studies [7], [8], [9] suggests that one third of the population have impaired voice production at some point in their lives (temporary) and further that the number of new patients with significant, long lasting voice problems (e.g. laryngectomees) are annually around 35, 000 in OECD countries.

Patients reduced to whispering have generally lost their pitch generation mechanism [1] through physiological blocking of vocal cord vibrations or, in pathological cases, blocking through disease or exclusion by an operation. Typical prostheses for voice impaired patients (esophageal speech [10], transoesophageal puncture (TEP) [11], and electrolarynx devices [12]) allow patients to regain limited speaking ability but do not generate natural sounding speech; at best their sound is monotonous or robotised [13], [14], [15], [16]. Additional drawbacks of traditional prostheses are difficulty of use and risk of infection from surgical insertion [17], [18]. Thus, within a speech processing framework, recent computational reconstruction methods (and particularly whispers to phonated speech) are aiming to regenerate natural sounding speech for aphonic and dysphonic individuals. Furthermore, comparing with traditional prostheses, these methods would be noninvasive and non-surgical.

Couple of methods are available for converting whispers to normal speech [19], [20], [21], [22], [23]. The driving idea of all these methods is based on the assumption of whispers are missing some acoustic and spectral features comparing with normal speech; hence, the problem of converting whispers to normal speech is formalised as a reconstruction issue [4], [24]. Through this approach, these methods aim to add or enhance missing or modified features and increase the signal similarity of whispers to normal speech. In general, these reconstruction methods can be classified into two major groups of training and non-training based methods. Utilising machine learning algorithms are the basis of training-based methods (whispers are mapped to the corresponding normal speech), while nontraining methods rely upon whisper enhancement and pitch regeneration.

These reconstruction methods (either training-based or nontraining) have different disadvantages such as problems in converting continuous speech (due to using phoneme switching) [20], being computationally expensive (due to using highly overlapped frames for spectral enhancement, or using jump Markov linear system for pitch and voicing parameters) [19], 


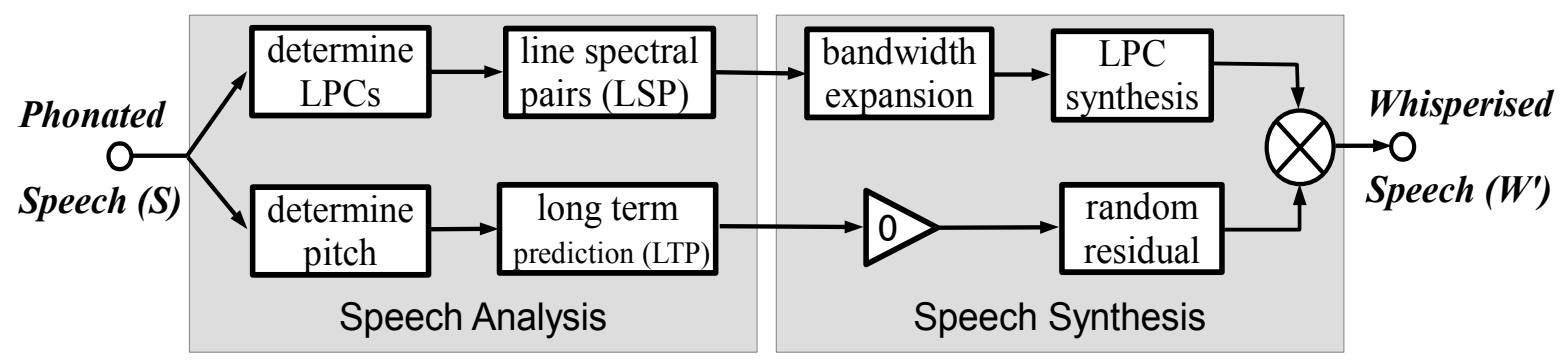

Fig. 1. Block diagram of generating whisperised speech from phonated speech.

[4], and more importantly lack of naturalness in regenerated output (due to simplified time alignment and spectral features assumptions) [21], [23]. In this paper, we focus on a trainingbased approach, and propose a novel reconstruction algorithm to improve the efficiency in phonated speech regeneration. In our algorithm, an intermediary layer called "artificial whisper" or "whisperised speech" is introduced to lessen the effect of inconsistent spectral features and time alignment between natural and whispered speech.

Section II explains whisperised speech while Section III addresses time alignment problem and describes our reconstruction algorithm using twin mapping models. The results are presented in Section IV and finally, the paper is concluded in Section V.

\section{WHISPERISED SPEECH}

Whispers and natural speech have different acoustic and spectral characteristics; the most significant physical characteristic of whispers is the absence of vocal cord vibration, resulting in missing pitch [25] and harmonics. Using a source filter model [26], exhalation can be identified as the source of excitation in whispered speech, with the shape of the pharynx adjusted to prevent vocal cord vibration in normal speakers [27]. The open glottis in whispers acts like a distributed excitation source [28] and the turbulent aperiodic noise can be seen as the primary excitation in whispered speech [27]. Whispered vowels and diphthongs also differ from fully phonated ones. Formant frequencies tend to be higher than in normal speech [2], particularly the first formant which shows the greatest difference between two kinds of speech.

Whisperised speech or artificial whisper is a whisper-like speech which is derived from normal speech by taking pitch off (i.e. eliminating periodic glottal excitation or removing long term prediction coefficients in standard source-filter model). The basic structure of analysis and synthesis parts employed in this paper for generating whisperised speech (W') from normal phonated speech $(\mathrm{S})$ is presented in Fig. 1.

In the analysis part, the phonated speech is first segmented into overlapped frames (50\% overlap and $15 \mathrm{~ms}$ duration for our configuration) and then linear predictive coding (LPC) analysis is performed on each frame to give a set of coefficients which are transformed into line spectral pairs (LSP). Finally, long-term prediction (LTP) filter provides pitch harmonics of the speech sample. In the speech synthesis part, LPC synthesis filter is used to reproduce the speech spectrum (to maintain formants), while making the LTP filter coefficients (pitch gain and therefore, pitch lags) equal to zero leads to pitch removal. The resultant pitch-less speech is defined as whisperised speech.

Being time aligned with natural speech and having similar spectrum (while sounds like whispers) are the main characteristics of whisperised speech. These features will be used in our proposed reconstruction algorithm to reduce the effect of time alignment and to give pitch variation in regenerated speech. The details of the approach are discussed in Section III.

\section{TRAINING-BASED RECONSTRUCTION}

\section{A. Time Alignment}

In training-based systems used for voice conversion, Gaussian mixture model (GMM)-based methods are state-of-theart at present [21], [23]. Similar to established learningtraining algorithms, the essential component of the trainingbased speech reconstruction process is also the extraction of particular features from the training inputs. In GMMbased systems, features are extracted from both natural speech and whispers, and then the relation (i.e. static and dynamic correspondence) between these features are found. Therefore, two parallel data sets of the same sentences are required, one in form of natural speech and the other in form of whispered speech; the more different two data sets are the more difficult is to map features. As discussed in Section II, natural speech and whispers are significantly different in terms of acoustical and spectral features; such substantial differences mainly affect the performance of the training-based reconstruction algorithms.

In the current systems, to adjust time durations and frame to frame alignment in training phase, a technique called dynamic time warping (DTW) is used [24] for the same utterances in whispered and phonated modes. DTW is originally employed for voice conversion in phonated speech and tries to match the two sentences based on their fundamental frequency and spectral similarities.

Therefore, DTW performance is well justified in voice conversion systems, where speech samples are normally phonated; i.e. both have components such as periodic excitation (fundamental frequency), obvious spectral envelope, formants, etc. 


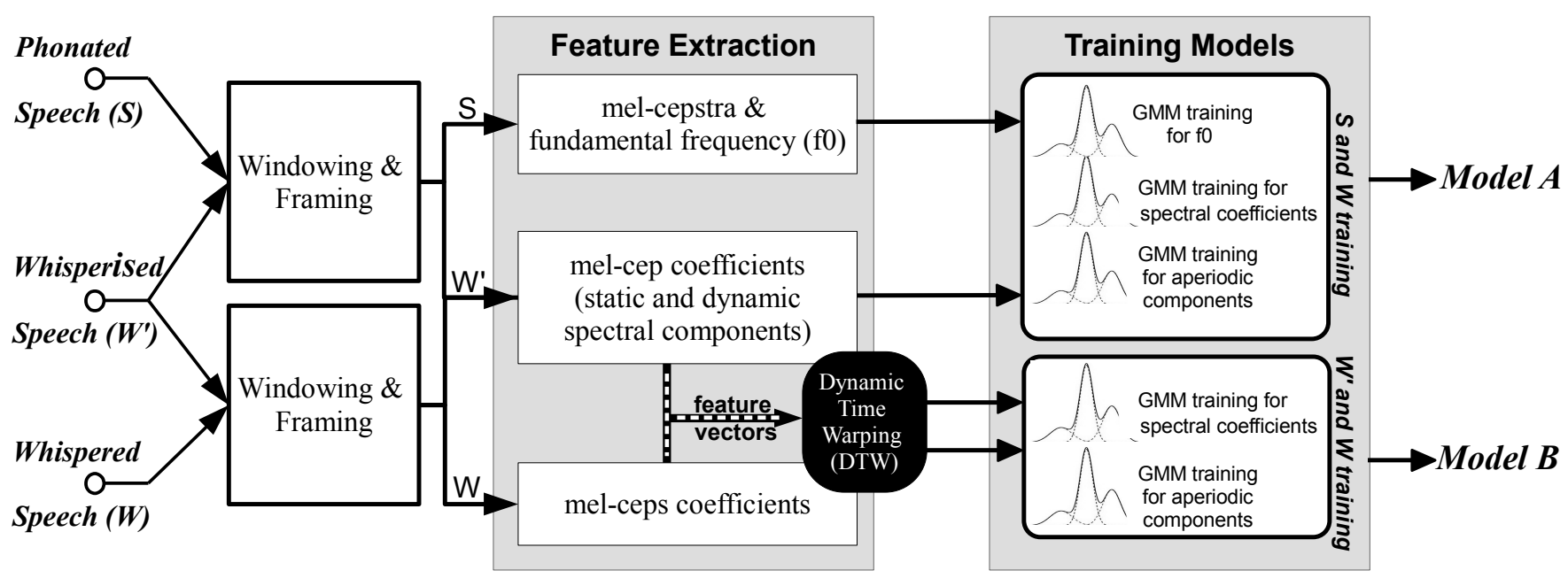

Fig. 2. Block diagram of our proposed method using two mapping models trained by whisperised speech.

On the other hand, DTW performance significantly reduces while working on unvoiced speech due to lack of fundamental frequency and noisy excitation, which leads to smooth spectrum and unclear formants.

Whisperised speech, as generated by the method proposed in II, is frame to frame time aligned with phonated speech; so by utilising this advantage in training phase and by introducing a twin GMM reconstruction algorithm, we can overcome the DTW limitation. In our method, we propose a twin mapping algorithm, which includes an intermediary layer to address the alignment problem in phonated and whispers pairs. To generate the intermediary layer, natural speech dataset is converted to whisperised speech dataset with the procedure described in Section II. The details of our algorithm using whisperised speech for training the system is discussed in the following subsection.

\section{B. Twin GMM}

In the current voice conversion systems based on GMM, voice features including mel-cepstrum coefficients and fundamental frequency (F0) are extracted using STRAIGHT [29] for each frame of whispered and phonated samples. Then, in an iterative process, DTW tries to align these two feature vectors, based on minimising the Euclidian distance between them.

As previously described, whispered speech not only takes longer duration than normal speech for pronouncing the same utterance but also lacks the fundamental frequency; therefore DTW cannot efficiently work on whispered samples due to missing some features in extracted vector by STRAIGHT. To address this problem, we insert whisperised speech as an intermediary layer between whispers and normal speech and train two mapping models: one for mapping from whispered to whisperised and one for mapping from whisperised to normal speech.

As previously discussed, whisperised speech have similar spectral features (except harmonics) and acoustic duration to normal speech, so time alignment (hence, DTW) is not required for such mapping model. This can partially address the time alignment issue between whispers and normal speech.

Figure 2 demonstrates the block diagram of our proposed method using whisperised speech (W') which is added as an intermediate state. In this method, first the whispered speech is converted to whisperised speech (through trained model $B$ ) and then the generated whisperised speech is converted to natural speech (through trained model $A$ ) with a regular GMM-based training system. The mapping model $A$ is trained based on whisperised speech and phonated speech; and the other mapping model (model $B$ ) is trained using pair of whisperised speech and whispered samples.

As described in Section II, whisperised speech is generated from the natural speech of the same utterance; hence samples are completely time aligned. The high level of similarity between two feature vectors in model $A$ improves feature matching. Furthermore, using whisperised speech and phonated speech for training the mapping model $A$ does not involve any time alignment process (i.e. DTW). With this training approach, the extracted features of whisperised speech and whispers are more similar to each other in comparison to whispered and phonated speech feature which is used in the current voice conversion systems. Therefore, comparing with the single GMM method, our proposed twin GMMs algorithm using whisperised speech can lead to a higher quality regenerated speech due to taking advantage of an efficient time alignment procedure. The analysis of this method and reconstruction result are discussed in Section IV.

\section{AnALYSIS AND RESUlts}

\section{A. Whisperised Speech}

Figure 3 shows time domain and spectrogram plots of a standard sentence from TIMIT corpus ("This was easy for us."). The sentence has been articulated in whispers (top) and spoken (middle) by the same speaker; then it has been 

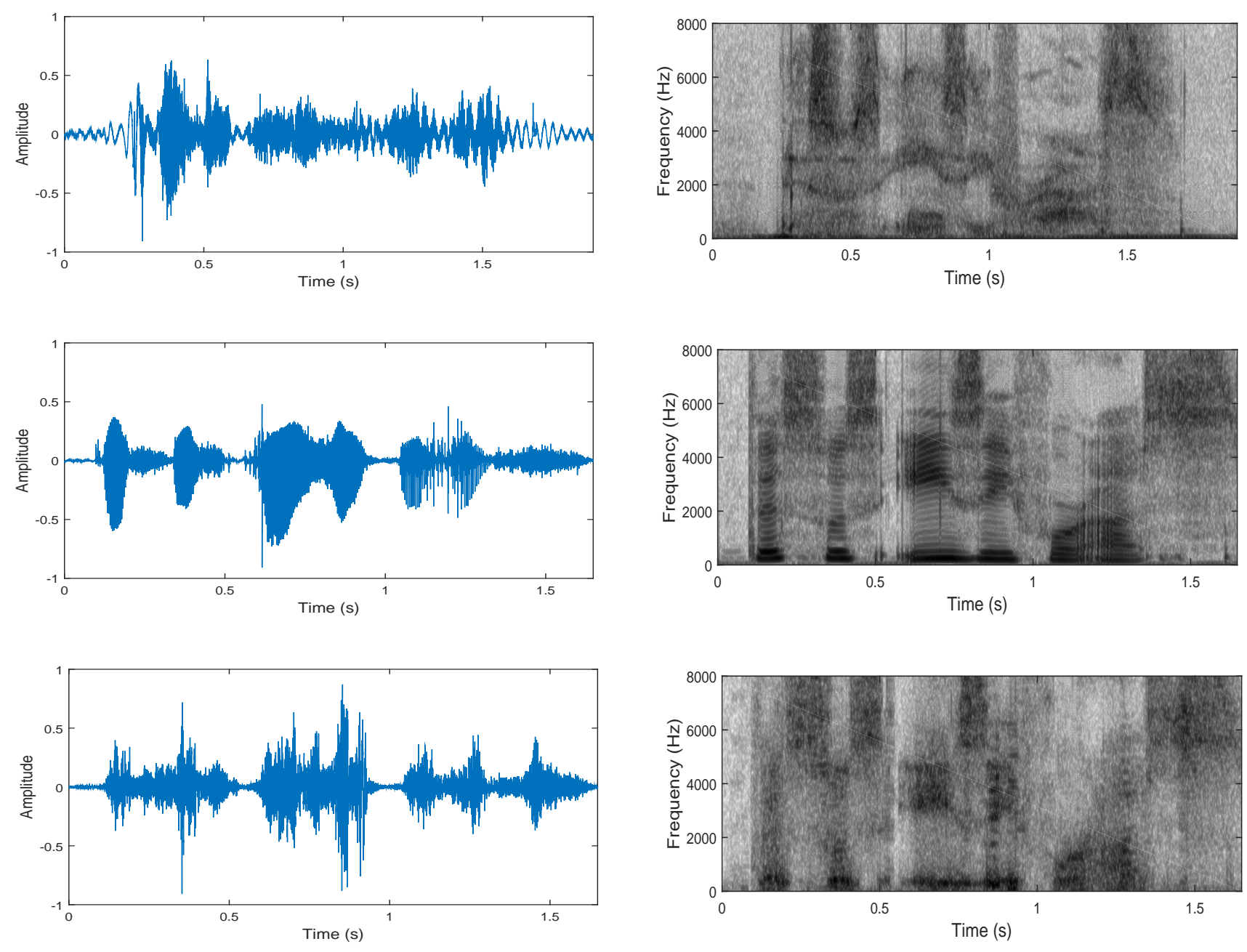

Fig. 3. Waveform and spectrogram plots of the sentence "This was easy for us." showing (top) whispered, (middle) spoken and (bottom) whisperised speech. All are amplitude normalised prior to plotting.

whisperised (bottom) through the method described in Section II. As illustrated in the figure, whispered (top) and spoken (middle) of a sentence are not time aligned and have different frame to frame durations. Hence, using these recordings as parallel utterance data for training GMM systems lead to the poor performance of DTW (and therefore degraded regenerated speech). On the other hand, as it can be seen in the Figure 3 , whisperised speech (bottom) is completely time aligned with spoken speech (middle). Furthermore, spectrogram of whisperised speech (bottom) resembles acoustic features of whispered speech (top) and this can make whisperised speech a reasonable choice to be used as parallel utterance data for training purposes.

\section{B. Phonated Speech Reconstruction}

Using the algorithm proposed in Section III-B, two models are trained relying upon whisperised speech in between. For the training datasets, 150 parallel whispered and spoken sentences recorded from North American speakers (20 persons) was obtained from wTIMIT corpus [30] and then whisperised speech dataset (through the method described in Section II) was generated accordingly.

As an example to demonstrate the efficiency of the proposed algorithm, waveforms and spectrograms are plotted for whispered, whisperised and reconstructed speech for the sentence "This was easy for us." in Figure 4: Whispered speech (top) is the input of the system, whisperised speech (middle) shows the output of whispers to whisperised model (mapping model $B$ ), and finally, reconstructed speech (bottom) displays the output of whisperised to phonated speech model (mapping model $A$ ) as the ultimate output of the system. As it is evident from the figure, the spectrogram of the reconstructed speech (bottom) shows phonated speech features: prominent formant bands, harmonics pertaining to fundamental frequency, and lower frequency energy distribution. This demonstrates the efficiency of the proposed algorithm in speech regeneration.

\section{CONClusion}

A method for whisper-to-speech reconstruction which relies upon twin mapping models was discussed in this paper. Our method makes use of an intermediary layer of whisperised 

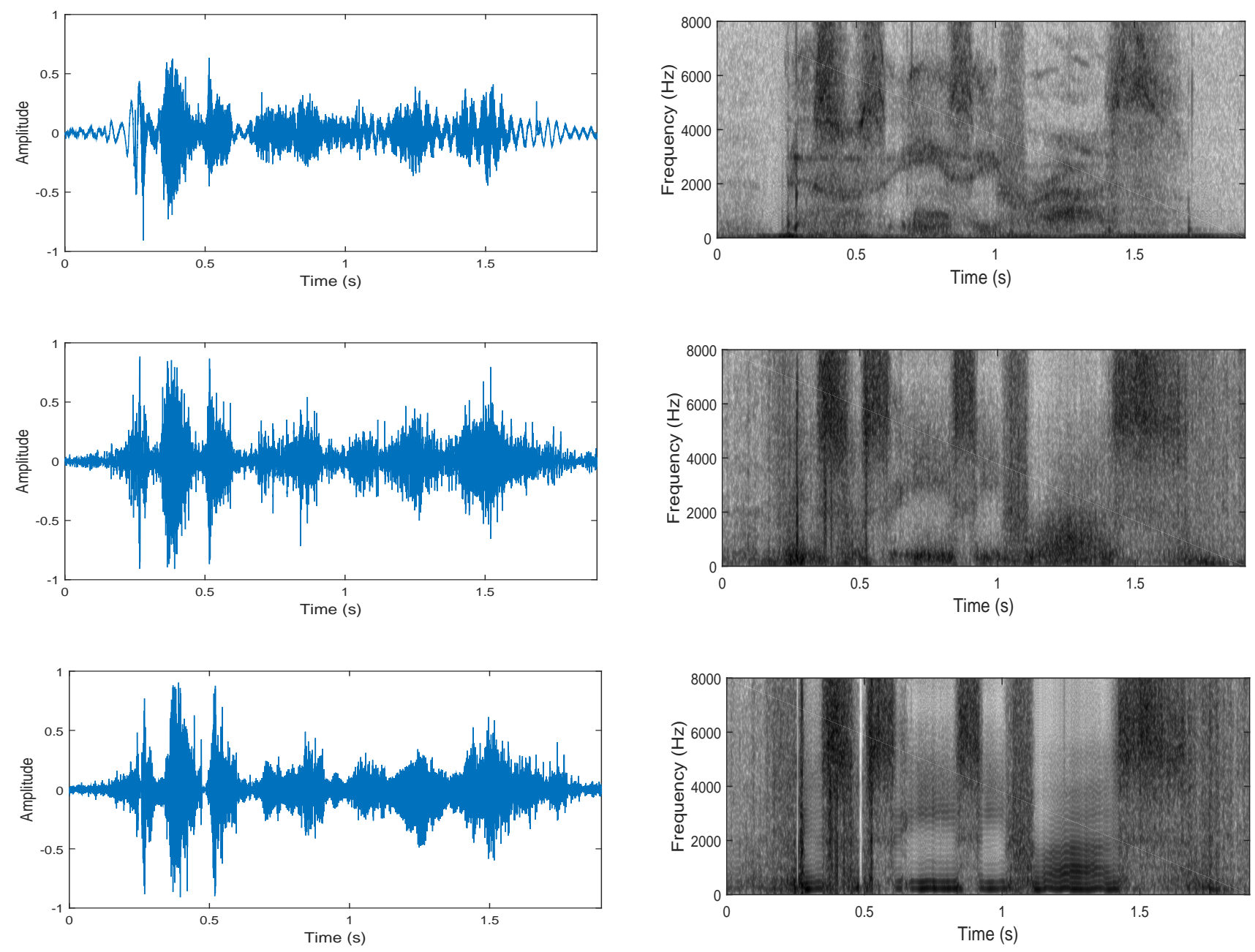

Fig. 4. Waveform and spectrogram plots of the sentence "This was easy for us." showing (top) whispered, (middle) whisperised and (bottom) reconstructed speech. All are amplitude normalised prior to plotting.

speech (artificial whisper) to address the alignment problem in phonated and whispered utterances which are basically used as parallel data for training GMM-based voice conversion systems.

Furthermore, the procedure for generating whisperised speech from normal speech was also described. Being time aligned with natural speech and having similar spectral features (while sounds like whispers) are the main characteristics of generated whisperised speech. Taking advantage of these features, the proposed reconstruction algorithm reduces the effect of time alignment and provides an efficient reconstruction technique.

\section{ACKNOWLEDGMENT}

The first two authors would like to thank the Unitec Research Committee (URC) for funding this project under Foci aligned funding 2015 (Grant Ref. RI14043).

\section{REFERENCES}

[1] R. Pietruch, M. Michalska, W. Konopka, and A. Grzanka, "Methods for formant extraction in speech of patients after total laryngectomy," Biomedical Signal Processing and Control, vol. 1, pp. 107-112, 2006.
[2] H. R. Sharifzadeh, I. V. McLoughlin, and M. J. Russell, "A comprehensive vowel space for whispered speech," Journal of Voice, vol. 26, no. 2, pp. e49-e56, 2012.

[3] I. McLoughlin, Applied Speech and Audio Processing. Cambridge: Cambridge University Press, 2009.

[4] H. R. Sharifzadeh, "Reconstruction of natural souding speech from whispers," Ph.D. dissertation, Nanyang Technological University, Singapore, 2012.

[5] N. P. Solomon, G. N. McCall, M. W. Trosset, and W. C. Gray, "Laryngeal configuration and constriction during two types of whispering," Journal of Speech and Hearing Research, vol. 32, pp. 161-174, 1989.

[6] V. C. Tartter, "Identifiability of vowels and speakers from whispered syllables," Perception and Psychophysics, vol. 49, pp. 365-372, 1991.

[7] S. R. Schwartz, S. M. Cohen, S. H. Dailey, R. M. Rosenfeld, and E. S. Deutsch, "Clinical practice guideline: hoarseness (dysphonia)," Otolaryngology Head and Neck Surgery, vol. 141, pp. S1-S31, 2009.

[8] L. B. Thomas and J. C. Stemple, "Voice therapy: does science support the art?" Communicative Disorders Review, vol. 1, pp. 49-77, 2007.

[9] L. O. Ramig and K. Verdolini, "Treatment efficacy: voice disorders," Journal of Speech Language and Hearing Research, vol. 41, pp. S10116, 1998.

[10] M. Azzarello, B. A. Breteque, R. Garrel, and A. Giovanni, "Determination of oesophageal speech intelligibility using an articulation assessment," Revue de laryngologie, otologie, rhinologie, vol. 126, pp. 327-334, 2005 . 
[11] V. Callanan, P. Gurr, D. Baldwin, M. White-Thompson, J. Beckinsale, and J. Bennet, "Provox valve use for post-laryngectomy voice rehabilitation," Journal of Laryngology and Otology, vol. 109, pp. 1068-1071, 1995.

[12] J. H. Brandenburg, "Vocal rehabilitation after laryngectomy," Archives of Otolaryngology, vol. 106, pp. 688-691, 1980.

[13] G. Culton and J. Gerwin, "Current trends in laryngectomy rehabilitation: A survey of speech language pathologists," Otolaryngology - Head and Neck Surgery, vol. 115, pp. 458-463, 1998.

[14] H. Liu, Q. Zhao, M. Wan, and S. Wang, "Enhancement of electrolarynx speech based on auditory masking," IEEE Transactions on Biomedical Engineering, vol. 53, pp. 865-874, 2006.

[15] E. A. Goldstein, J. T. Heaton, J. B. Kobler, G. B. Stanley, and R. E. Hillman, "Design and implementation of a hands-free electrolarynx device controlled by neck strap muscle electromyographic activity," IEEE Transactions on Biomedical Engineering, vol. 51, pp. 325-332, 2004.

[16] G. A. Gates, W. Ryan, J. C. Cooper, G. F. Lawlis, E. Cantu, T. Hayashi, E. Lauder, R. W. Welch, and E. Hearne, "Current status of laryngectomee rehabilitation: I. results of therapy," American Journal of Otolaryngology, vol. 3, pp. 1-7, 1982.

[17] R. Hillman, M. Walsh, G. Wolf, and S. Fisher, "Functional outcomes following treatment for advanced laryngeal cancer. part 1 . voice preservation in advanced laryngeal cancer. part ii. laryngectomy rehabilitation: the state-of-the-art in the va system," Annals of Otology, Rhinology and Laryngology, vol. 107, pp. 1-27, 1998.

[18] H. R. Sharifzadeh, I. V. McLoughlin, and F. Ahmadi, Lecture Notes in Electrical Engineering. Springer, 2010, ch. Speech rehabilitation methods for laryngectomised patients, pp. $597-607$.

[19] R. W. Morris and M. A. Clements, "Reconstruction of speech from whispers," Medical Engineering and \& Physics, vol. 24, pp. 515 - 520, 2002.

[20] H. R. Sharifzadeh, I. V. McLoughlin, and F. Ahmadi, "Reconstruction of normal sounding speech for laryngectomy patients through a modified celp codec," IEEE Transactions on Biomedical Engineering, vol. 57, pp. 2448-2458, 2010

[21] T. Toda, M. Nakagiri, and K. Shikano, "Statistical voice conversion techniques for body-conducted unvoiced speech enhancement," IEEE Transactions on Audio, Speech, and Language Processing, vol. 20, no. 9, pp. $2505-2517,2012$

[22] I. V. McLoughlin, H. R. Sharifzadeh, S. Tan, J. Li, and Y. Song, "Reconstruction of phonated speech from whispers using formantderived plausible pitch modulation," ACM Transactions on Accessible Computing, vol. 6, no. 4, pp. 12:1-12:21, 2015

[23] J. Li, I. V. McLoughlin, L. Dai, and Z. Ling, "Whisper-to-speech conversion using restricted boltzmann machine arrays," Electronics Letters, vol. 50, no. 24, pp. 1781 - 1782, 2014.

[24] T. Toda, A. W. Black, and K. Tokuda, "Voice conversion based on maximum-likelihood estimation of spectral parameter trajectory," IEEE Transactions on Audio, Speech, and Language Processing, vol. 15, pp. 2222-2235, 2007

[25] V. C. Tartter, "Whats in a whisper?" Journal of the Acoustical Society of America, vol. 86, pp. 1678-1683, 1989.

[26] G. Fant, Acoustic Theory of Speech Production, 2nd ed. The Hague: Mouton, 1960.

[27] I. B. Thomas, "Perceived pitch of whispered vowels," Journal of the Acoustical Society of America, vol. 46, pp. 468-470, 1969.

[28] K. N. Stevens, Acoustic Phonetics. Cambridge, MA: The MIT Press, 1998.

[29] H. Kawahara, I. Masuda-Katsuse, and A. D. Cheveigne, "Restructuring speech representations using a pitch-adaptive timefrequency smoothing and an instantaneous-frequency-based f0 extraction," Speech Communication, vol. 27, no. 3, pp. 187 - 207, 1999.

[30] B. P. Lim, "Computational differences between whispered and nonwhispered speech,” Ph.D. dissertation, University of Illinois, 2010. 